\title{
Evolution of magnetic properties during tempering
}

\author{
Ville Akujärvi ${ }^{1}$ - Tord Cedell ${ }^{1}$. Oleksandr Gutnichenko ${ }^{1}$ - Matias Jaskari ${ }^{2} \cdot$ Mats Andersson $^{1}$
}

Received: 24 May 2021 / Accepted: 25 November 2021 / Published online: 6 December 2021

(c) The Author(s) 2021

\begin{abstract}
Quality control in heat treatment of steel is often conducted after the treatment. Failure to confine within the specified range of mechanical properties may lead to wasted energy and production resources. Performing quality control in-line in the heat treatment process allows for early detection and possibility to react to changes in the process. The prospects of utilizing the change in the electromagnetic (EM) properties of steel, as means for quality control, are investigated in this paper. The focus is on the tempering process of hardened SS2244 (42CrMoS4) steel. The tempering takes the hardness of the steel from approximately $600 \mathrm{HV}$ down to around $400 \mathrm{HV}$. The EM signature of the steel is recorded during the tempering process. This is later compared with results from more traditional means of material characterization, such as laser scanning microscopy, $\mathrm{X}$-ray diffractometry and Vickers microhardness measurement. This initial study shows clear indications of precise detection of the hardness through EM properties during the tempering process of selected material.
\end{abstract}

Keywords In-line measurement · Quality control $\cdot$ Electromagnetic properties $\cdot$ Tempering

\section{Introduction}

Tempering is a heat treatment technique in which the goal is to reduce the hardness of hardened steel and thereby increase its toughness. The tempering process is dependent on both process temperature and time as well as material composition. Empirical relations, such as Holloman-Jaffe [1], between hardness and tempering time and temperature are usually used to determine the appropriate process parameters. These give good guidelines, but perhaps not the sufficiently correct value for any given steel if the data available is insufficient [2].

To ensure the performance of the heat-treated steel, or finished product, is as specified, quality control is traditionally performed after the tempering process. If the performance is substandard, the process parameters can be adjusted. This procedure is fine while tuning the process but will become a problem if something unexpected occurs. In the best case, the steel can be put through the hardening process again and

Ville Akujärvi

ville.akujarvi@iprod.lth.se

1 Production and Materials Engineering, Lund University, Lund, Sweden

2 FMT-group, Kerttu Saalasti Institute, University of Oulu, Oulu, Finland then re-tempered. In the worst case, this leads to scrapping of substandard products. Performing quality control early in the process could potentially catch mistakes before the product has become too much refined. Ideally quality control would occur in-line within the heat treatment process, with a nondestructive method. Traditional methods for mechanical testing of steels are hard to incorporate in-line, since they are designed to work in controlled environments. Measurement of any quantitative or qualitative value of, for example, hardness must be performed indirectly, as to not affect the finished product.

Inductive sensors have been used to measure properties indirectly within a variety of different areas. For instance, inductive sensors have been used to measure temperature in domestic induction cooktops [3] as well as in harsh environments $[4,5]$.

Inductive methods have also been used in quality control. Detection of cracks and voids in, for example, welded joints are made possible by inducing eddy currents in the target area and then either measuring the leakage flux $[6,7]$ or detecting local deviations in the thermal response [8-10]. Eddy current sensors have also been used in other applications, such as detecting defects buried inside parts made with additive manufacturing [11] and as a method for automated measurement of the thickness of metallic plates [12]. For grinding processes, Barkhausen noise has been used to monitor the process and detect thermal damage [13]. 
It has also been shown that the depth of decarburization can also be measured by utilizing the difference in the electromagnetic (EM) response at different depths [14].

In this paper, the aim is to investigate the correlation between the variation of microstructure of low-alloyed steel and its EM properties during the tempering process. Since the steel hardness is one of the most sensitive properties to the changes in materials microstructure, the obtained data allow estimation of the applicability (sensitivity and reliability) of the proposed technique for in-line measurements of hardness indirectly through EM properties and/or its monitoring during heat treatment. This paper focuses on tempering, although other heat treatment processes of steels could also be suitable to utilize with this technique. The concept could be realized as a stand-alone sensor, as means for feedback control from the process, or being a part of the system, if induction heaters are used. The analysis of main factors influencing the magnetic properties and EM measurements of steels are introduced in Sect. 2.

The material selected for this study is SS2244 (42CrMoS4), which is commonly used in industrial applications and has been well studied by researchers. As such, there are plenty of articles about the influence of different types of heat treatment on the microstructure and phase transformation, e.g., [15-19]. This allows for greater focus on the changes in magnetic properties during heat treatment and their correlation with the microstructure and phase change in the material in the present study.

\section{Electromagnetic-mechanical coupling}

There are numerous factors which contribute to the magnetic properties of steels, including chemical composition, impurities, strain, temperature, crystal structure and crystal orientation. The magnetic properties can be categorized into two groups: structure-insensitive and structure-sensitive [20], as summarized in Table 1 . In addition, the remanence $\left(B_{r}\right)$ is also considered to be structure-sensitive [21]. In a broad sense, this gives an indication to what underlying mechanism may affect a certain magnetic property. As tempering alters the target materials' microstructure, it is reasonable to

Table 1 Magnetic properties divided into structure-sensitive and structure-insensitive groups [20]

\begin{tabular}{ll}
\hline Structure-insensitive & Structure-sensitive \\
\hline Saturation magnetization & Permeability \\
Curie point & Coercive force \\
Magnetostriction at saturation & Hysteresis loss \\
Crystal anisotropy constant & \\
\hline
\end{tabular}

assume that the magnetic properties listed in the structuresensitive column will change during the process.

There is generally no single cause that determines the magnetic properties of a material. The main contributors to magnetic properties are the composition, crystal structure and crystal orientation. Depending on composition, different heat treatment techniques may be used to alter the crystal structure and hence the mechanical properties such as hardness and yield strength. A mechanically hard steel is generally also magnetically hard. This means that processes aimed at making steels mechanically softer, more ductile, would also restore magnetic softness to the same steel. Magnetically hard materials are often characterized with larger coercivity $\left(H_{c}\right)$, which requires higher magnetic field strength $(H)$ to affect the state of magnetization, e.g., reverse the direction of magnetization or to demagnetize.

Alloying elements affect the overall magnetic properties of a given steel, both by which combination of alloying elements and by which quantities they are used. Some elements will affect the overall magnetic properties to a great extent, while others show no overall significant impact. Finding openly published magnetic properties for any given steel grade is hard, if not impossible. Bozorth [20] has compiled the work of numerous researchers, where the most common binary and ternary ferromagnetic alloys are considered. His work is mostly aimed at applications related to permanent magnets or flux conductors, which is to say that the presented quantities of alloying elements are more related to magnetic components rather than structural components. Alloying elements commonly found in structural steels which have a somewhat marked effect of the magnetic properties include carbon $(\mathrm{C})$, chromium $(\mathrm{Cr})$, molybdenum (Mo) and nickel (Ni), while manganese $(\mathrm{Mn})$ and silicon (Si) have little to no effect. Phosphorus (P) and sulfur (S) are considered impurities and as such known to affect the magnetic properties [20].

Impurities and other imperfections, such as inclusions, dislocations and voids in the crystal structure, hinder the domain wall motion of nearby domains. Domain walls are generally situated in a local energy minimum, i.e., small applied field strength would cause minor movement of the domain wall around its minimum and as the field is removed the domain wall would fall back to its original resting position. Imperfections are in this case larger energy maximums, which require large field strengths to push the domain walls past it. In fact, the domain walls prefer to bend around the site of imperfection, given the field strength is not large enough to push it completely over the site. Whenever a large enough field strength is applied to warrant a domain wall motion beyond an imperfection, the domain wall would find a new local energy minimum at the other side of the imperfection when the applied field is removed. The effect of the 
imperfections on the bulk material manifests as decreased initial relative permeability $\left(\mu_{r}\right)$ and increased $H_{c}$ [22].

Steel sample under applied mechanical stress will show different magnetic properties compared to a relaxed sample. This effect is also different, depending on whether the magnetic properties are measured along the stress axis or orthogonal to it. Applied tensile stress on a sample with positive magnetostriction will favor the magnetic domains oriented in the same direction as the stress, i.e., parallel or antiparallel to the stress axis. This will make the favored domains grow in size, at the expense of the unfavored, and hence become more easily magnetizable in the direction of the tensile stress [23].

The relative permeability of a ferromagnetic material changes with temperature, becoming paramagnetic as the Curie temperature $\left(T_{c}\right)$ is reached. The behavior is different depending on the field strength applied to the specimen. For high field strengths, $\mu_{r}$ decreases slightly with increasing temperature at low temperatures. The decrease becomes larger as the temperature increases until the material becomes paramagnetic. For low field strength, this behavior is quite different. The change in $\mu_{r}$ is subtle at low temperature, remaining mostly constant. The relative permeability starts to increase rapidly when the temperature approaches $T_{c} . \mu_{r}$ reaches its maximum value just below $T_{c}$, after which it rapidly descends to unity for higher temperatures. This characteristic behavior is associated with the low magnetic anisotropy energy at temperature close to $T_{c}$ [20].

Crystal orientation of ferromagnetic material is rarely oriented randomly in space, instead they have a preferred orientation, called crystallographic texture. The texture depends on the shape and process used to form the bulk product. The preferred orientation is close to parallel to, for example, the rolling direction for sheet metal. Grain-oriented electrical steel uses the preferred orientation from rolling to its advantage to produce a better magnetic flux conductor commonly used in electrical machines and power transformers [23].

\section{Experimental work}

\subsection{Procedure}

The steel SS2244 (42CrMoS4) is chosen as the target material for the measurement as a conventional construction material that usually undergoes heat treatment (both quenching and tempering) before use. The standard chemical composition is shown in Table 2. The ring-shaped steel samples, see Sect. 3.3 for ring dimension, were austenitized at $840{ }^{\circ} \mathrm{C}$ and quenched in oil, resulting in a hardness $600 \mathrm{HV}$ prior to tempering. The tempering temperature was selected to $560{ }^{\circ} \mathrm{C}$, providing relatively large change in the mechanical properties of the steel as compared to quenched material; see
Table 2 Standard chemical composition for SS2244 (42CrMoS4)

\begin{tabular}{ll}
\hline Element & Weight [\%] \\
\hline $\mathrm{C}$ & $0.38-0.45$ \\
$\mathrm{Si}$ & $0.10-0.40$ \\
$\mathrm{Mn}$ & $0.60-0.90$ \\
$\mathrm{P}$ & $0.035(\max )$ \\
$\mathrm{S}$ & $0.02-0.04$ \\
$\mathrm{Cr}$ & $0.90-1.20$ \\
$\mathrm{Mo}$ & $0.15-0.30$ \\
$\mathrm{Fe}$ & Rest \\
\hline
\end{tabular}

Table 3. The tempering time was left as a process parameter to control the outcome of the heat-treated samples.

The experimental work is divided into two parts: One is related to characterization of the samples' microstructure and microhardness, and one for the EM properties of steel samples at different tempering intensity. As the result of tempering is dependent on both time and temperature, the ovens were preheated to $560^{\circ} \mathrm{C}$ to provide a consistent starting point for the samples for both the mechanical properties and the EM properties. The tempering intensity is controlled by the tempering time in this study as the tempering temperature remains constant.

The samples for the estimation of variation of steel's microstructure and microhardness were extracted from quenched ring-shaped samples. The ring was cut in roughly 8-mm segments, using CBN cutting wheel and water flood cooling during the cut. The resulting roughly cube-shaped samples, with a side length of $8 \mathrm{~mm}$, were tempered in the preheated oven. The samples were extracted one by one after different amount of tempering time $(\mathrm{t}=1,2,4,6,8$, $10,12,14,16,18,25 \mathrm{~min})$ and left to cool down in room temperature.

As for the EM measurement, the procedure is somewhat different. The same sample is subjected to the entire tempering cycle, because the interest in this study lies in finding the correlation of the in-line measured EM signature of the sample to the finished tempered sample. The EM properties were measured at specific times, trying to match the ones from the other measurement part as well as take supplementary measurements in between where possible.

Table 3 Comparison of mechanical properties between tempered and quenched sample of $42 \mathrm{CrMoS} 4$ according to material specification

\begin{tabular}{lll}
\hline Properties & Tempered & Quenched \\
\hline UTS [MPa] & 1200 & 1900 \\
$0.2 \%$ YS [MPa] & 1100 & 1550 \\
Elongation [\%] & 12 & 3 \\
Impact strength $\left[\mathrm{J} / \mathrm{cm}^{2}\right]$ & 65 & 30 \\
\hline
\end{tabular}




\subsection{Hardness and microstructure}

Assessment of the mechanical properties was performed through the measurements of Vickers microhardness with a loading of $1 \mathrm{~kg}$ and duration of $10 \mathrm{~s}$ (THV-30MDX). The microhardness was measured 8-10 times in different places on the polished sample surface, and mean values were taken into account.

For the microstructural study, the samples were first mounted and then polished mechanically to mirror-finish using $1 \mu \mathrm{m}$ colloidal silica suspension. The samples were then etched with $2 \%$ Nital etchant for revealing the microstructure, and investigation was done with laser scanning microscope (LSM) (Keyence VX-200).

The changes of the samples' microstructure due to the heat treatment was also studied with X-ray diffractometry (XRD) in $\mathrm{CuK} \alpha$ radiation (STOE STADI MP). The relative concentration of carbon in martensite phase at different tempering times was estimated by calculation of the interplanar spacing of martensite lattice in (110) plane by the equation

$d^{2}=\frac{a^{2}}{h^{2}+k^{2}+\frac{l^{2} a^{2}}{c^{2}}}$

where $c / a=1+0.467 p$ lattice tetragonality, $\mathrm{p}$ carbon content in the martensite [24].

\subsection{Electromagnetic properties}

Depending on what the aim is with measurement of EM properties, the frequency at which these are measured may be selected differently. A low frequency is generally a good choice if the fundamental EM properties of a material are of interest. Here, the measured magnetic hysteresis loss $\left(W_{h}\right)$ can be correlated with microstructural hardness of the sample. For higher frequencies, in the order of several $\mathrm{kHz}$, other factors tend to drive the losses. The bulk of the induced currents are located closer to the exterior (closest to the excitation winding) of the sample due to the skin effect, increasing the eddy current losses significantly, when the frequency is increased. The hysteresis losses are still present in the sample but now obscured by eddy current losses, and separation between the losses is all but simple to establish. Of course, parameters such as sample size should be considered when selecting the frequency. For very thin samples, a higher frequency is more relevant to get appropriate readings. Higher frequencies could also be useful when trying to mimic a real process, e.g., induction heating. In this case, one would also have to revise the sample and coil geometry to something analogous to the process. The result would then also be more related to inductance with some effective $\mu_{r}$ parameter, rather than the fundamental $B-H$ relationship.
The EM properties are measured on a toroid (see Fig. 1) sample with the size $80 \mathrm{~mm}$ outer diameter, $64 \mathrm{~mm}$ inner diameter and $8 \mathrm{~mm}$ height. The toroid is wound, like a transformer, with a primary and a secondary winding. The turns ratio is 30:30, i.e., there is the same amount of turns on the primary as the secondary winding. The insulation of the wire used in the winding is rated to withstand $1000{ }^{\circ} \mathrm{C}$, allowing measurements to be made at elevated temperatures, without running the risk of short-circuiting the windings.

To measure the EM properties, the primary side is excited with low-frequency high-amplitude current, which induces a voltage across the secondary winding. The primary current corresponds to the magnetic field strength $(H)$, giving according to Ampere's circuital law [25]

$H=\frac{N_{p} I_{e x}}{l_{e}}$

where $N_{p}$ is the number of turns on the primary, $I_{e x}$ is the excitation current and $l_{e}$ is the effective magnetic path length of the toroid. According to Faradays law of induction [25], the time integral of the induced voltage on the secondary side corresponds to magnetic flux density $(B)$ with

$B=\frac{1}{N_{s} A_{e}} \int_{0}^{T} U d t$

where $N_{s}$ is the number of turns on the secondary side, $A_{e}$ is the effective magnetic cross-sectional area of the toroid and $U$ is the induced voltage drop. $A_{e}$ and $l_{e}$ are used to compensate for the toroid geometries which have a large difference between the inner and outer diameter. These formulas recalculate the geometry to match the mean path perceived by the magnetic field. See [26] for formulas for calculation of $l_{e}$ and $A_{e}$. However, these could be approximated with the average circumference and the cross-sectional area and still be below one percent error in the approximation with the toroid size used in this study. $B$ and $H$ give the constitutive relation [25]

$B=\mu_{0} \mu_{r} H$

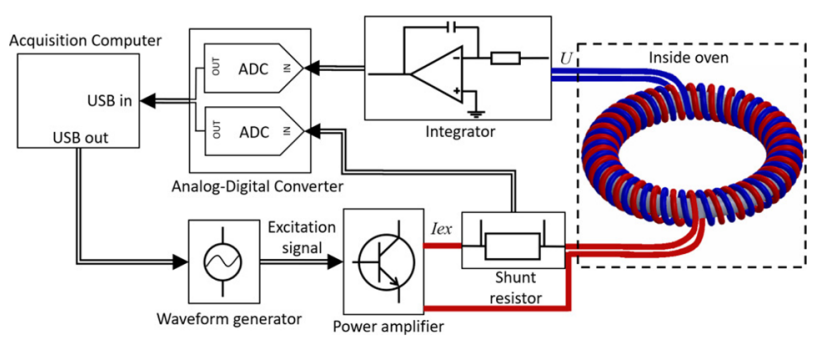

Fig. 1 Illustration of the measurement setup, highlighting the main components. The windings are colored to differentiate between excitation current $I_{e x}$ (red) and induced voltage $U$ (blue) 
where $\mu_{0}$ is the vacuum permeability $\left(4 \pi 10^{-7} \mathrm{H} / \mathrm{m}\right)$ and $\mu_{r}$ is the relative permeability. This relation is often illustrated with the $B-H$ curve, as shown in Fig. 2. The figure illustrates other measurable quantities as well, such as the coercivity $\left(H_{c}\right)$, remanence $\left(B_{r}\right)$ and the hysteresis loss $\left(W_{h}\right)$ (enclosed area of the $B-H$ curve).

The Fischer Feritscope FMP30, hereinafter referred to as Feritscope, was used as a complement to the EM measurements. This device is normally used to measure ferrite content in weld beads for parts made of austenitic stainless steels. At its core, the Feritscope induces a magnetic field into the target material and measures the EM response of the material, likely in the terms of magnetic permeability [27]. As austenitic phases are paramagnetic, the device can detect the degradation of the permeability, i.e., a fully austenitic steel would have a $\mu_{r}$ close to one, while a steel which is a mixture of ferromagnetic phases (such as martensite) and austenite would have a higher $\mu_{r}$. The response shown by the Feritscope is, through its internal software and calibration reference, converted to a value corresponding to a ferrite content as indicated by the reference calibration. The ferrite content reading given by the Feritscope can be converted into a martensite content, given that a calibration curve has been established. This type of study has been performed for
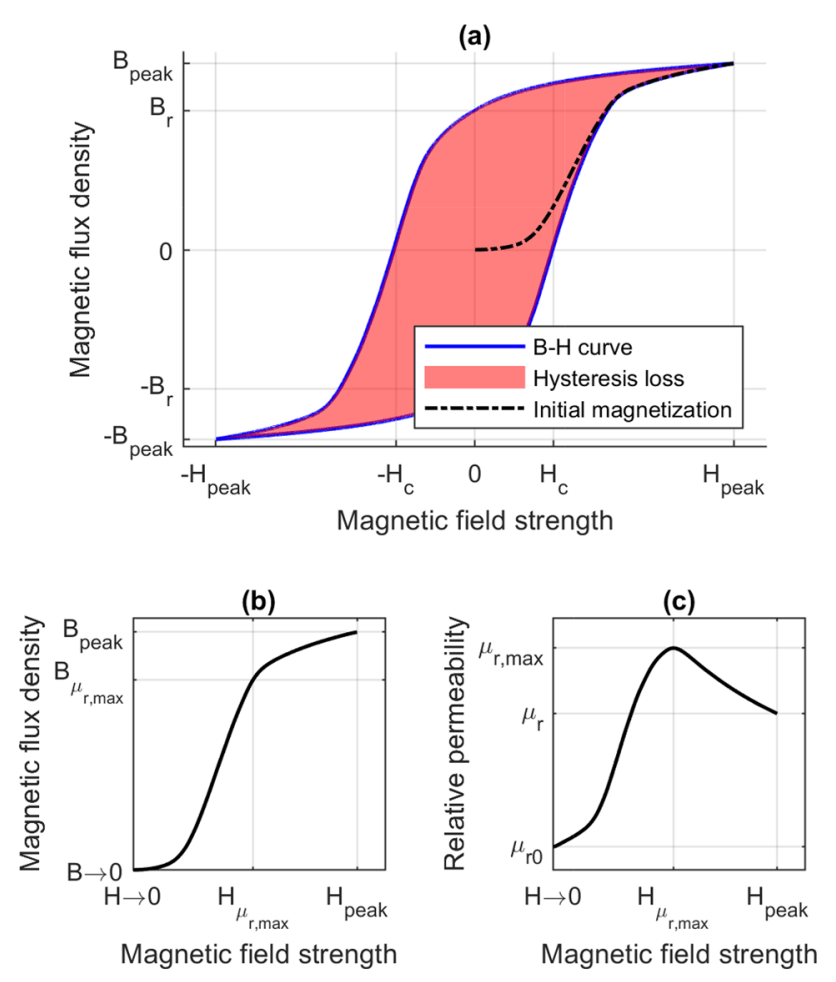

Fig. 2 Illustration of a (a) generic $B-H$ curve, (b) initial magnetization and (c) the corresponding relative permeability, where $\mu_{r 0}$ is the initial relative permeability and $\mu_{r, \max }$ is the maximum relative permeability austenitic steel grades AISI 304 and 301LN [28], where the authors found that a simple linear correction factor could be applied to get the martensite content from the ferrite content reading given by the Feritscope. It cannot be assumed that this correction factor applies directly to other steel grades, such as the one used in this study. No actual effort has been put into finding appropriate correction factor for this specific steel. But it is still interesting as a point of reference of a method closely related to the proposed approach. A subset of the samples from the hardness and microstructure analysis was selected to be measured using the Feritscope $(t=0,1,2$, $4,12,25 \mathrm{~min})$. Each of the samples was measured 10 times at arbitrary locations to get an average reading.

\section{Results and discussion}

\subsection{Hardness and microstructure}

Measured microhardness of the samples at different tempering times is illustrated in Fig. 3a. LSM images of the microstructure are also shown on the diagram (Fig. 3b-e). It is evident that decreasing character of the microhardness curve with an increase in tempering time is related to the degradation of martensite during the process as demonstrated by the LSM images (see Fig. 3).

XRD measurements were performed to analyze the martensite transformation during tempering. A general view of the XRD spectra at different tempering times is presented in Fig. 4a.

Analysis of the XRD spectra shows that all significant peaks correspond to the martensite lattice in different direction (Fig. 4a). No austenite phase and no doublets or triplets of the peaks were detected at the given test and measurement conditions $\left(2 \theta=20-110^{\circ}, \Delta \theta=0.05^{\circ}\right.$, dwell $\left.10 \mathrm{~s}\right)$.

A more detailed measurement was performed for two samples-a reference sample (only quenched) and a sample tempered for $25 \mathrm{~min}$ (see Fig. 4b). The measurement condition: diapason $2 \theta=30-110^{\circ}, \Delta \theta=0.02^{\circ}$, dwell $15 \mathrm{~s}$. The precise measurements confirm the previous conclusions regarding the present phases, but allow detecting the shift of the peaks in the (110) plane that apparently is related to the diffusion of carbon atoms from the martensite lattice (see embedded image in Fig. 4b).

Further measurements were focused on the peak shift in the diapason of $2 \theta$ angles between $42^{\circ}$ and $48^{\circ}$ with the measurement condition $\Delta \theta=0.01^{\circ}$ and dwell $30 \mathrm{~s}$. The measurements were performed for the assessment of the change in carbon content in the martensitic lattice.

The results, as illustrated in Fig. 5, show that the peaks' intensity increases, while the width decreases with increasing tempering time, a behavior which is related to the diffusion of carbon. Besides that, the peak displacement is quite 
Fig. 3 Correlation between (a) microhardness and microstructure of LSM images for different tempering times: (b) 0 min, (c) 4 min, (d) 12 min and (e) $25 \mathrm{~min}$

(a)

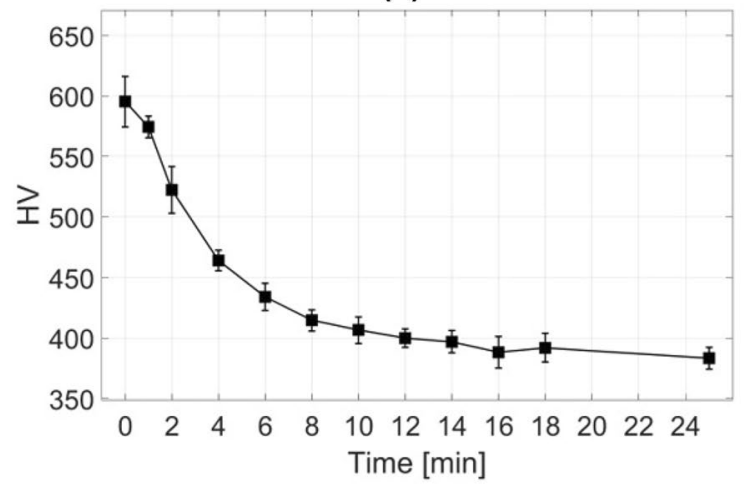

(b)

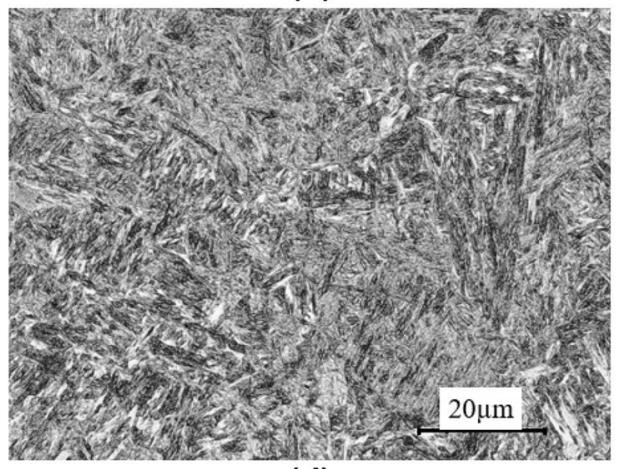

(d)

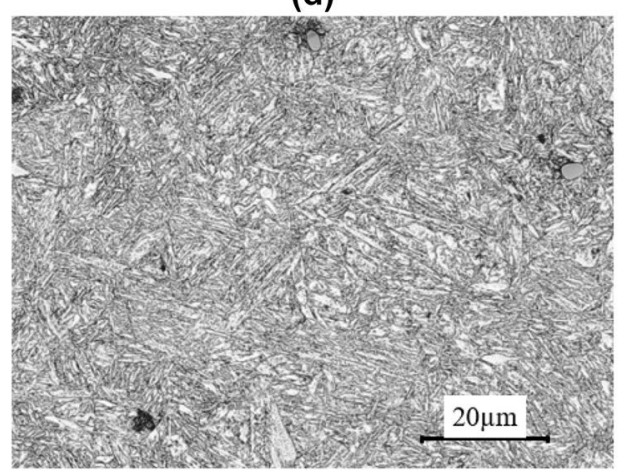

(c)

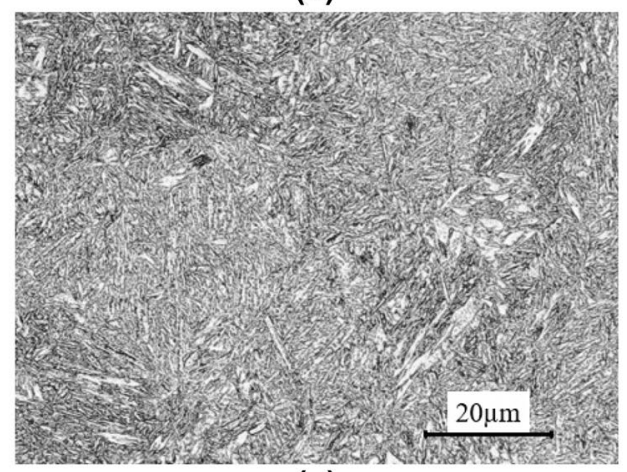

(e)

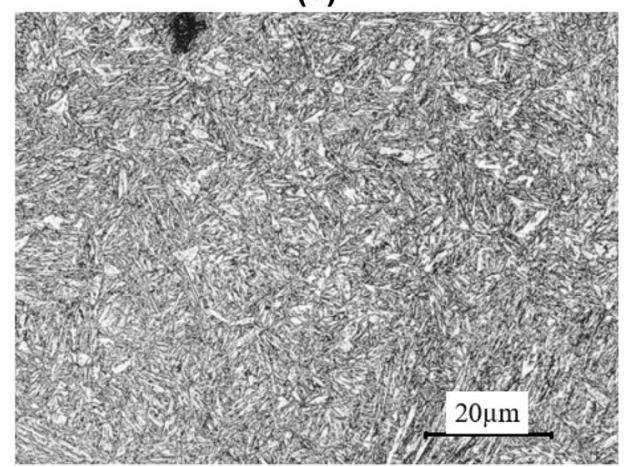

significant at small tempering times and becomes negligible when tempering time exceeds 10 minutes, which correlates quite well with microhardness curve (Fig. 3a).

The measured peak shifts at different tempering times allows, using Eq. (1), estimating the change of relative carbon content in the martensitic phase, presented in Fig. 6. From the results in Fig. 6, it is evident that it is in good agreement with obtained data for microhardness.

Both XRD and LSM results indicate fully martensitic structure. The lacking evidence of retained austenite might be due to a high martensite start temperature [29]. For the $42 \mathrm{CrMo} 4$, the martensite start temperature has been measured to the range of $310-331^{\circ} \mathrm{C}[17,18]$. Carbides are not resolved by optical microscopy, nor does the XRD analysis show any presence of carbides. It is believed that carbides in fact exist, but to such a small extent that it drowns in noisy area of the XRD spectra. Sackl et al. [18] found chromium-rich carbides only by using transmission electron microscopy (TEM) in their study on quenched $42 \mathrm{CrMo} 4$ [18] as well as cementite in their complementary study on tempered 42CrMo4 [19]. The relatively short tempering times used in this study might be a contributing factor for the apparent absence of carbides, i.e., not facilitating enough time for the carbide precipitation and growth kinetics.

\subsection{Electromagnetic properties}

The $B-H$ curves measured during the tempering process are shown in Fig. 7 where the initial magnetization curves from 


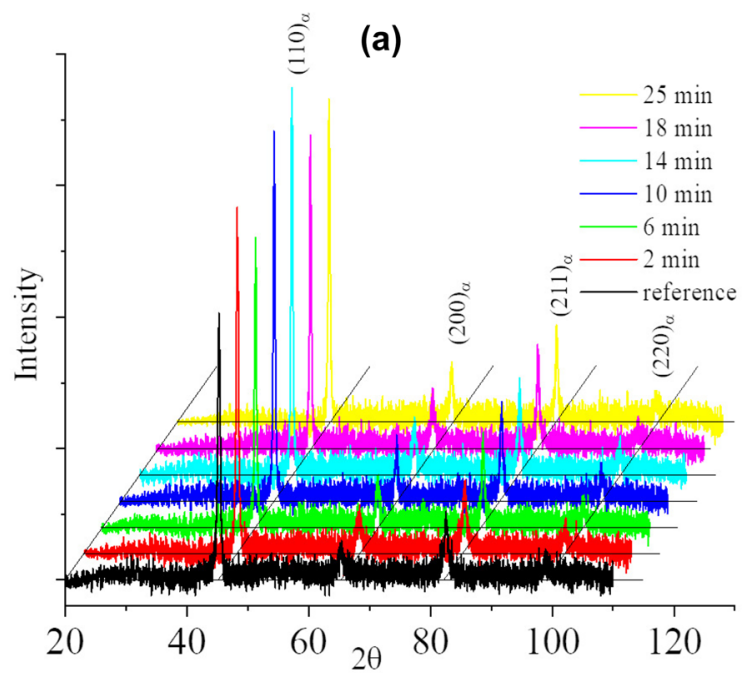

(b)

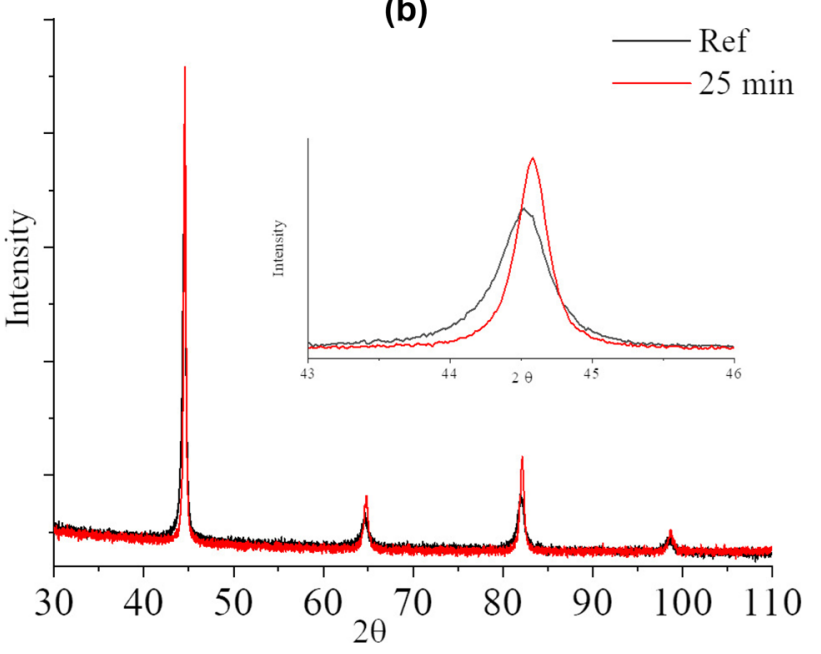

Fig. 4 (a) General view of the XRD spectra of the samples at different tempering times and (b) more detailed view of the XRD spectra for reference sample and sample tempered for $25 \mathrm{~min}$

the demagnetized state are excluded. Here, the curves are decreasing in size with increasing tempering time, with a more rapid pace in the beginning.

Since it is a bit cumbersome to read specific values from the cluster of $B-H$ curves, these are summarized in Fig. 8. From here, it is easier to identify what happens at different tempering times. Time zero corresponds to the electromagnetic properties in its hardened state, i.e., prior to inserting it into the oven. The first measurement at heated state occurs at 4 minutes, corresponding to the time it took to insert the sample into the oven with the windings attached and connecting it to the measurement system. The remaining measurements were taken approximately 2 minutes apart. The time necessary for one measurement was around $6 \mathrm{~s}$, excluding postprocessing and time to write acquired data to disk for later analysis. The long acquisition time allowed

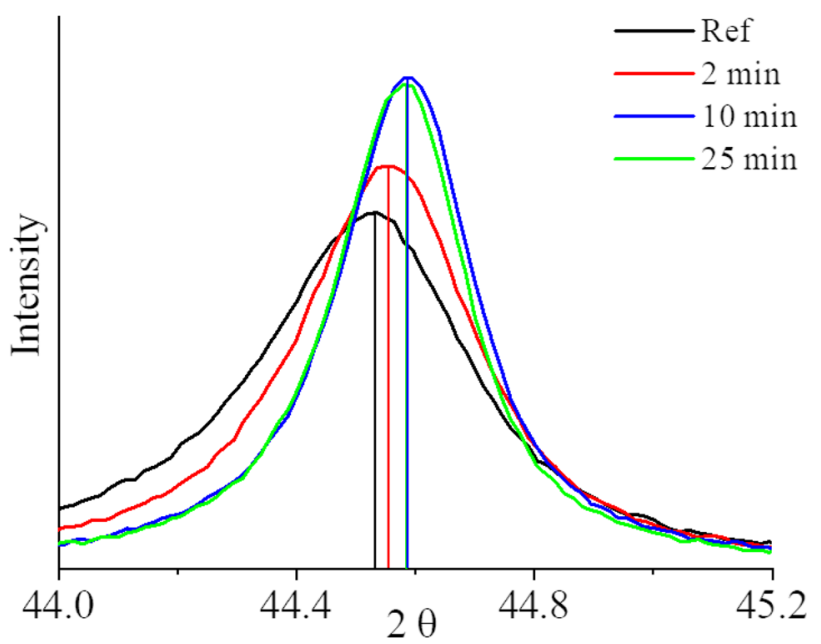

Fig. 5 Peak shift for different tempering times

for 3 periods at an excitation frequency of $0.5 \mathrm{~Hz}$. Multiple periods allow for effectively erasing any remaining magnetization from previously applied field during the first period. The excitation current through the primary winding was held constant throughout the measurement series, corresponding to an average peak magnetic field strength of $5.362 \mathrm{kA} / \mathrm{m}$, with a standard deviation of $0.763 \mathrm{~A} / \mathrm{m}$.

The most prominent changes can be seen for the $H_{c}$ and $W_{h}$, which are approximately decreased by a factor of two during the first 10 minutes of measurements, followed by a smaller change of $B_{r}$. The variation of $\mu_{r}$ and $B_{\text {peak }}$ is not consistent with the change in material properties, rather caused by the variation in the applied magnetic field strength.

The structure-sensitive properties will generally improve with larger grains, less dislocations, reduced debris in the

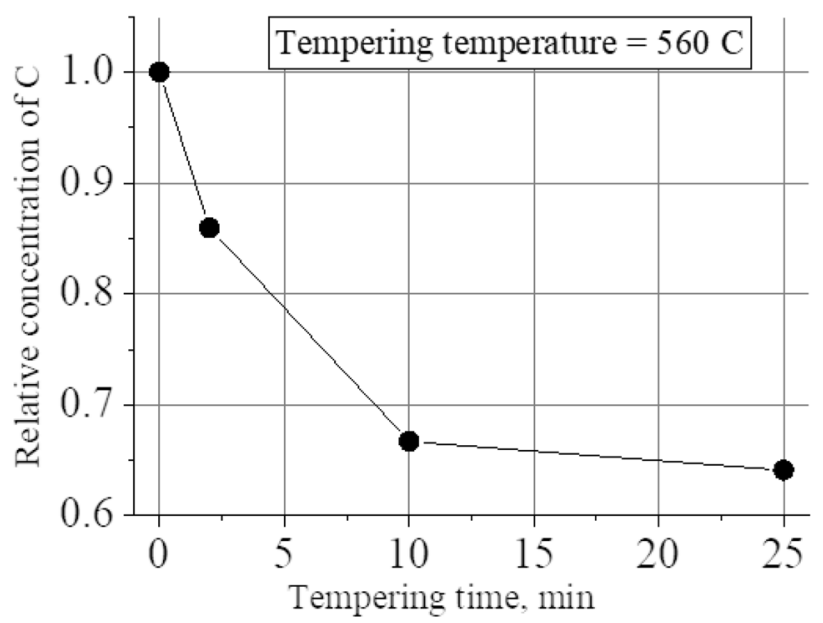

Fig. 6 Change of relative concentration of carbon in martensite during tempering 


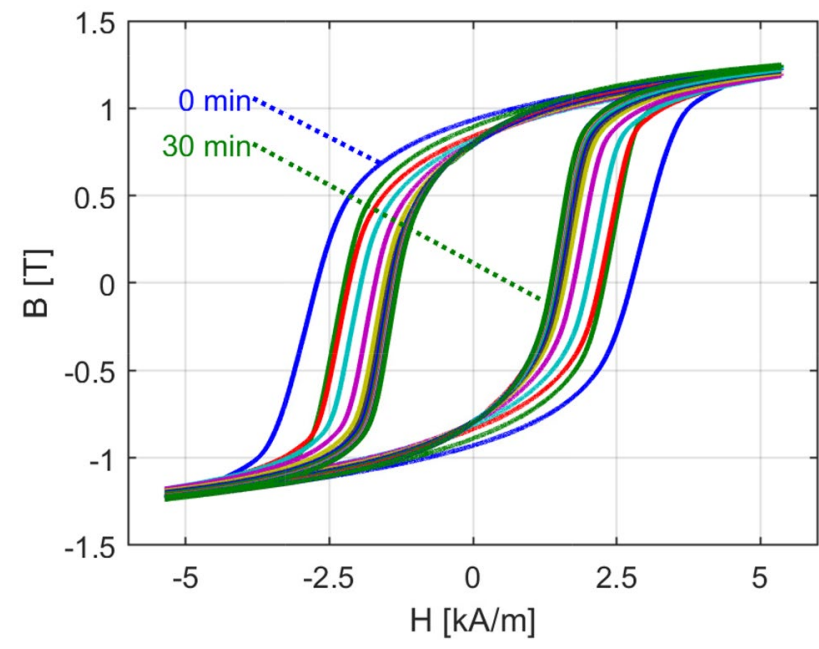

Fig. 7 Collection of $B-H$ curves measured during tempering. The $B-H$ loop is decreasing in size as the tempering time is increasing

grain boundaries and reduced residual stresses. This is observable in the EM measurements, i.e., both the hysteresis loss and coercivity decrease as the tempering time increases (Fig. 8d-e). It should be noted that $\mu_{r}$ does not appear to change with any significance during the tempering (Fig. 8b). This lack of response in the measured relative permeability is likely due to the choice of its representation, i.e., $\mu_{r}$ at peak applied field strength. As the $B-H$ loop of ferromagnetic material is nonlinear, there is no single value of $\mu_{r}$ which describes the entire loop (see illustration in Fig. 2c). If instead the initial magnetization was analyzed, there would probably be noticeable difference in the initial $\mu_{r}$ and/or maximum $\mu_{r}$. The initial $\mu_{r}$ is inherently hard to acquire due to the accuracy required in both producing and measuring in low magnetic field strengths. As described by Rayleigh law, see for instance [23], the permeability converges to a constant value as the field strength goes toward $0 \mathrm{~A} / \mathrm{m}$. The maximum $\mu_{r}$ is on the other hand easier to capture since it would generally appear at field strengths in the vicinity of $H_{c}$. To get reliable measurement of the maximum $\mu_{r}$, one would have to perform a demagnetizing cycle in between each successive measurement. However, this was not performed to allow for measurements in more rapid succession.

The results from both microstructure characterization and EM measurements show good correlation between microhardness and coercivity. The results from both measurements are plotted alongside each other in Fig. 9 for easy comparison. The first 10 minutes show the most prominent response, with the highest gradient, for both cases. Beyond the first 10 minutes, the response flattens out, only showing small changes with increasing tempering time. The microstructure analysis suggests that the EM response is linked to the transformation of martensite grains due to the diffusion (a)

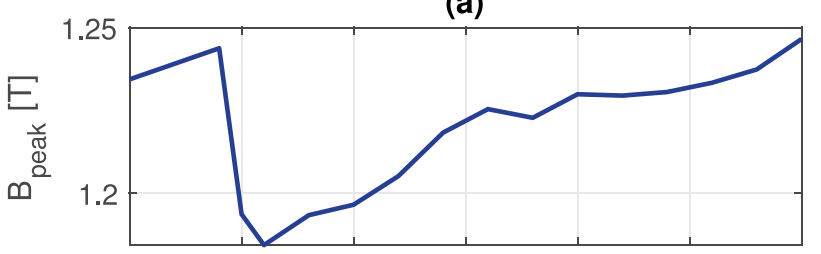

(b)

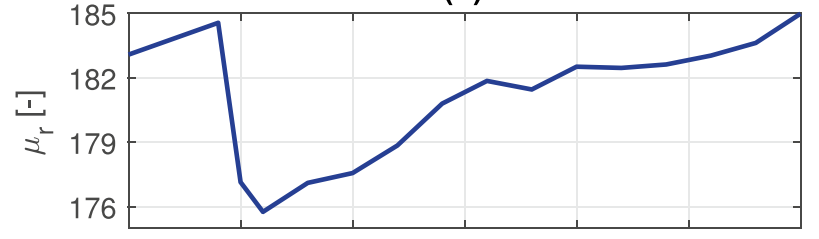

(c)

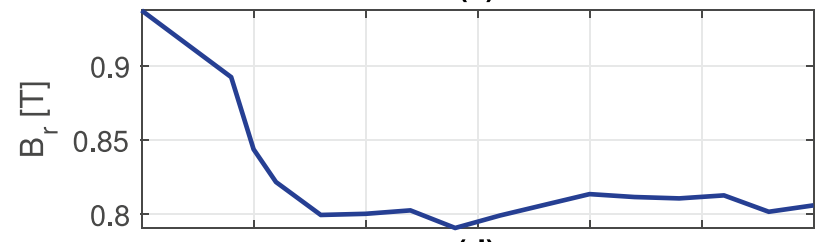

(d)
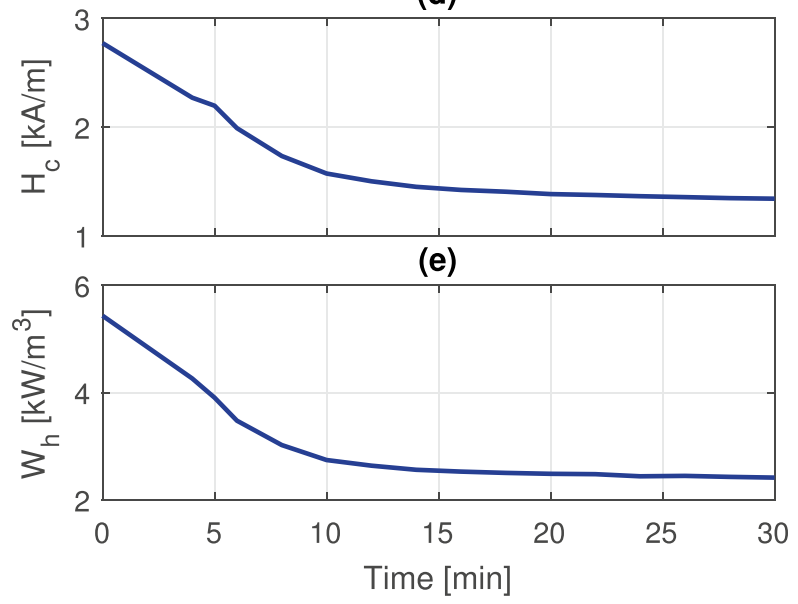

Fig. 8 Collection of discrete values from the $B-H$ curves, with $H_{\text {peak }}$ $=5.36 \mathrm{kA} / \mathrm{m}$. The figure includes (a) peak magnetic flux density, (b) relative permeability at $H_{\text {peak }}$ and $B_{\text {peak }}$, (c) remanence, (d) coercivity and (e) hysteresis loss. Note that figures (a)-(e) share the same $\mathrm{x}$-axis scale

of carbon atoms and relieving stresses of the martensite lattice (see Figs. 3 and 5).

The presence of carbides is known to affect the magnetic properties of ferromagnetic material in a negative manner, e.g., by decreasing the permeability and increasing the coercivity [30]. However, $H_{c}$ is steadily decreasing which would suggest that the main tempering mechanism is related to recovery rather than precipitation of carbides [31]. For longer tempering times, the recovery process might no longer be dominant or to some extent counteracted by carbide precipitation and growth, resulting in the reduced reduction rate of $H_{c}$. 


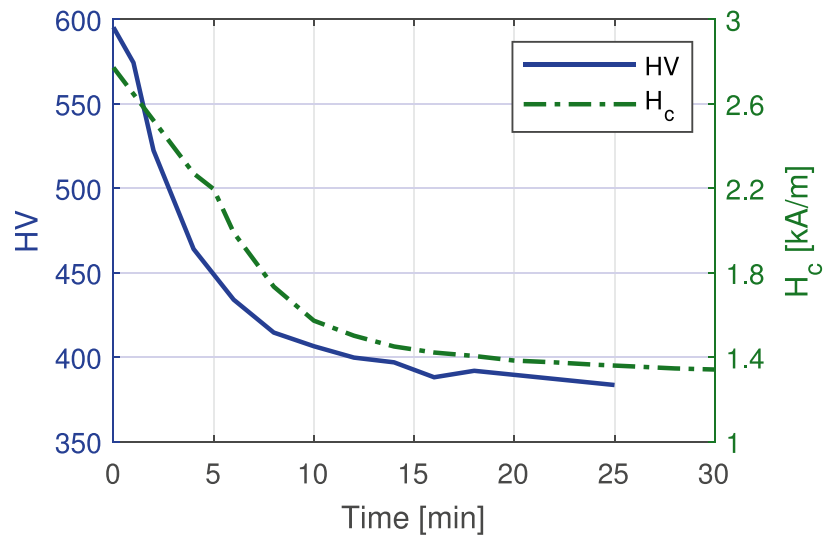

Fig. 9 Comparison between $\mathrm{HV}$ and $H_{c}$

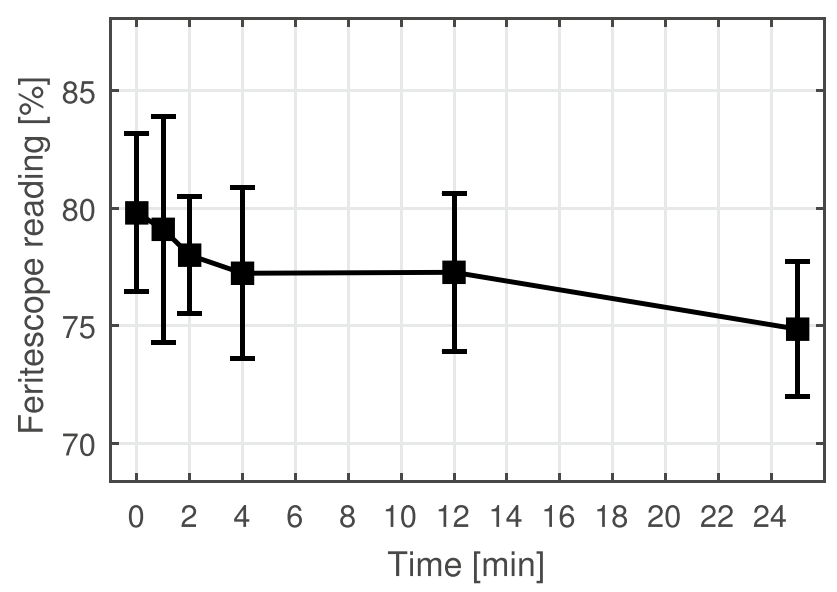

Fig. 10 Ferrite content measured with Feritscope. Squares indicate the mean value, and the vertical lines indicate the width of the standard deviation

The Feritscope reading shows a small decrease in ferrite content with increasing time, when looking at the mean values of the individual measurement for each sample (see Fig. 10). However, the error bound is quite high, rendering the results impractical for this purpose. Given the results from microstructure analysis, the lack of response from the Feritscope is understandable since no other phases than martensite appear to exist in the samples.

\section{Conclusions}

This initial study showed that during the tempering of lowalloyed steel $42 \mathrm{CrMoS} 4$ the needlelike structure of martensite of quenched steel transforms, by diffusion of carbon atoms, to tempered martensite with rounded needles/grains. Despite small changes in the material's microstructure, its microhardness decreases by more than a third during the first ten minutes, making it possible to detect the changes via measurement of its EM properties. The change in coercivity and hysteresis loss shows the most prominent EM response for the tempering process, decreasing by approximately a factor of two from its hardened state. The change in remanence showed a similar trend, while the change was not as prominent. This correlation between microhardness and EM properties is quite promising for further applications in quality monitoring and control systems on the industrial level.

The method used to measure the EM properties in this paper is feasible for laboratory work but problematic when considering the practical implementation in a real industrial process. To make use of this in a real process, the change in EM properties of a bulk material needs to be translated to other quantities which are more easily measurable. For the processes using induction heating, this could potentially be realized using the heating coil. For other heating types, one would have to use a separate inductive sensing head, much like the Feritscope. The results from the Feritscope measurements show no significant response to the tempering process. The error bounds are too large in comparison with the overall change to give any clear conclusion with regards to microstructural or microhardness changes. In all fairness, this is likely due to that the device is operated outside of the range of its intended application, or calibration.

As the investigation focused on only one steel grade, the results are not necessarily applicable directly on any other steel grade. However, the general behavior is believed to manifest in a similar manner in most ferromagnetic steels. Depending on the chemical composition and microstructure, the magnitude of the response might vary.

\section{Future work}

In the next phase, the study will continue with a wider range of different steel grades, to get a broader perspective of applicability of this type of measurement. The sensitivity of this type of measurement with regards to chemical composition is also of interest, e.g., to study how steel grades with similar chemical compositions would affect the EM signature.

Furthermore, a practical realization of this type of measurement should be conducted. This in a semi-industrial scale, preferably together with an induction heating process.

Acknowledgements The present work was carried out with support from Vinnova in project Induction heating with Structural feedback (InStruct), as well as within the Sustainable Production Initiative (SPI) - a cooperation between Lund University and Chalmers University of Technology.

Author Contributions Ville Akujärvi contributed to conceptualization, formal analysis, funding acquisition, investigation, methodology, 
resources, validation, visualization, and writing-original draft. Tord Cedell helped in conceptualization, funding acquisition, investigation, methodology, validation, and writing-review and editing. Oleksandr Gutnichenko performed formal analysis, investigation, methodology, validation, visualization, and writing-review and editing. Matias Jaskari was involved in investigation, validation, visualization, and writing-review and editing. Mats Andersson contributed to conceptualization, funding acquisition, methodology, project administration, supervision, and writing - review and editing.

Funding Open access funding provided by Lund University. The research was made possible through funding from Vinnova in project InStruct—Induction heating with Structural feedback (2018-04292).

\section{Declarations}

Conflicts of interest/Competing interests The authors declare that they have no conflicts of interest.

Open Access This article is licensed under a Creative Commons Attribution 4.0 International License, which permits use, sharing, adaptation, distribution and reproduction in any medium or format, as long as you give appropriate credit to the original author(s) and the source, provide a link to the Creative Commons licence, and indicate if changes were made. The images or other third party material in this article are included in the article's Creative Commons licence, unless indicated otherwise in a credit line to the material. If material is not included in the article's Creative Commons licence and your intended use is not permitted by statutory regulation or exceeds the permitted use, you will need to obtain permission directly from the copyright holder. To view a copy of this licence, visit http://creativecommons.org/licenses/by/4.0/.

\section{References}

1. Hollomon JH, Jaffe LD (1945) Time-temperature relations in tempering steel. Trans Am Inst Min Metall Eng 162:223-249

2. Canale L, Yao X, Gu J, Totten G (2008) A historical overview of steel tempering parameters. Int J Microstruct Mater Prop 3. https://doi.org/10.1504/IJMMP.2008.022033

3. Franco C, Acero J, Alonso R, Sagues C, Paesa D (2012) Inductive sensor for temperature measurement in induction heating applications. IEEE Sensors J 12(5):996-1003. https://doi.org/10.1109/ JSEN.2011.2167226

4. Varpula T, Sepp H (1993) Inductive noise thermometer: Practical realization. Rev Sci Instruments 64(6):1593-1600. https://doi.org/ 10.1063/1.1144031

5. Kisner R, Britton CL, Jagadish U, Wilgen JB, Roberts M, Blalock TV, Holcomb D, Bobrek M, Ericson MN (2004) Johnson noise thermometry for harsh environments. In: 2004 IEEE Aerospace Conference 4:2586-2596

6. Goktepe M (2001) Non-destructive crack detection by capturing local flux leakage field. Sensors Actuators A: Phys 91(1-2):70-72. https://doi.org/10.1016/S0924-4247(01)00511-8

7. Sophian A, Tian GY, Zairi S (2006) Pulsed magnetic flux leakage techniques for crack detection and characterisation. Sensors Actuators A: Phys 125(2):186-191. https://doi.org/10.1016/j.sna.2005.07.013

8. Oswald-Tranta B (2007) Thermo-inductive crack detection. Nondestruct Test and Evaluation 22(2-3):137-153. https://doi.org/10. 1080/10589750701448225

9. Noethen M, Wolter K, Meyendorf N (2010) Surface crack detection in ferritic and austenitic steel components using inductive heated thermography. In: 33rd Int Spring Seminar on Electron Technol, pp 249-254. https://doi.org/10.1109/ISSE.2010.5547297
10. Weekes B, Almond DP, Cawley P, Barden T (2012) Eddy-current induced thermography-probability of detection study of small fatigue cracks in steel, titanium and nickel-based superalloy. NDT \& E Int 49:47-56. https://doi.org/10.1016/j.ndteint.2012.03.009

11. Du W, Bai Q, Wang Y, Zhang B (2018) Eddy current detection of subsurface defects for additive/subtractive hybrid manufacturing. Int J Adv Manuf Technol 95(9):3185-3195. https://doi.org/10. 1007/s00170-017-1354-2

12. Nebair H, Cheriet A, El Ghoul IN, Helifa B, Bensaid S, Lefkaier IK (2018) Bi-eddy current sensor based automated scanning system for thickness measurement of thick metallic plates. Int $\mathbf{J}$ Adv Manuf Technol 96(5-8):2867-2873. https://doi.org/10.1007/ s00170-018-1753-z

13. Tnshoff HK, Karpuschewski B, Regent C (1999) Process monitoring in grinding using micromagnetic techniques. Int $\mathrm{J}$ Adv Manuf Technol 15(10):694-698. https://doi.org/10.1007/s001700050121

14. Hao XJ, Yin W, Strangwood M, Peyton AJ, Morris PF, Davis CL (2008) Off-line measurement of decarburization of steels using a multifrequency electromagnetic sensor. Scr Mater 58(11):10331036. https://doi.org/10.1016/j.scriptamat.2008.01.042

15. Zafra A, Peral LB, Belzunce J, Rodriguez C (2019) Effects of hydrogen on the fracture toughness of $42 \mathrm{CrMo} 4$ steel quenched and tempered at different temperatures. Int J Pres Ves Pip 171:34 50 https://doi.org/10.1016/j.ijpvp.2019.01.020

16. Thakare AS, Butee SP, Kamble KR (2020) Improvement in Mechanical Properties of $42 \mathrm{CrMo} 4$ Steel Through Novel Thermomechanical Processing Treatment. Metallogr Microstruct 9(5):759-773 https://doi.org/10.1007/s13632-020-00684-9

17. Feng J, Frankenbach T, Wettlaufer M (2017) Strengthening $42 \mathrm{CrMo} 4$ steel by isothermal transformation below martensite start temperature. Mater Sci Eng A-Struct Mater Prop Microstruct Process 683:110-115. https://doi.org/10.1016/j.msea.2016.12.013

18. Sackl S, Leitner H, Zuber M, Clemens H, Primig S (2014) Induction Hardening vs Conventional Hardening of a Heat Treatable Steel. Metall Mater Trans A 45a(12):5657-5666 https://doi.org/ 10.1007/s11661-014-2518-4

19. Sackl S, Zuber M, Clemens H, Primig S (2016) Induction Tempering vs Conventional Tempering of a Heat Treatable Steel. Metall Mater Trans A 47a(7):3694-3702 https://doi.org/10.1007/s11661-016-3534-3

20. Bozorth RM (1993) Ferromagnetism. Wiley

21. Jiles DC (1988) Review of magnetic methods for nondestructive evaluation. NDT Int 21(5):311-319. https://doi.org/10.1016/03089126(88)90189-7

22. Jiles DC, Atherton DL (1984) Theory of ferromagnetic hysteresis (invited). J Appl Phys 55(6):2115-2120. https://doi.org/10.1063/1. 333582

23. Cullity BD, Graham CD (2009) Introduction to magnetic materials, 2nd edn. IEEE/Wiley, Hoboken, N.J

24. Gorelik SS, Skakov YA, Rastorguev LN (1994) X-ray and Electron-Optical Analysis, 3rd edn. MISIS, Moscow

25. Haus HA, Melcher JR (1989) Electromagnetic fields and energy. Prentice Hall, Englewood Cliffs, N.J

26. Boehm A, Hahn I (2014) Measurement of magnetic properties of steel at high temperatures. In: 40th Annu Conf Ind Electron Soc (IECON), IEEE, pp 715-721 https://doi.org/10.1109/IECON.2014.7048579

27. Hecker SS, Stout MG, Staudhammer KP, Smith JL (1982) Effects of strain state and strain rate on deformation-induced transformation in 304 stainless-steel: Part I. magnetic measurements and mechanical-behavior. Metall Trans A: Phys Metall Mater Sci 13(4):619-626. https://doi.org/10.1007/Bf02644427

28. Talonen J, Aspegren P, Hanninen H (2004) Comparison of different methods for measuring strain induced alpha '-martensite content in austenitic steels. Mater Sci Technol 20(12):1506-1512. https://doi.org/10.1179/026708304x4367 
29. Krauss G (2012) 5 - Tempering of martensite in carbon steels. In: Phase Transformations in Steels, Vol 2. Woodhead Publishing https://doi.org/10.1533/9780857096111.1.126

30. Jiles DC (1988) Magnetic-Properties and Microstructure of AISI1000 Series Carbon-Steels. J Phys D Appl Phys 21(7):1186-1195 https://doi.org/10.1088/0022-3727/21/7/022

31. Kumar H, Mohapatra JN, Roy RK, Joseyphus RJ, Mitra A (2010) Evaluation of tempering behaviour in modified $9 \mathrm{Cr} 1 \mathrm{Mo}$ steel by magnetic non-destructive techniques. J Mater Process Technol 210(4):669-674 https://doi.org/10.1016/j.jmatprotec.2009.12.003

Publisher's Note Springer Nature remains neutral with regard to jurisdictional claims in published maps and institutional affiliations. 